\title{
Family-Wise Error Rate
}

National Cancer Institute

\section{Source}

National Cancer Institute. Family-Wise Error Rate. NCI Thesaurus. Code C64215.

A statistical method used for multiple testing corrections to control the expected proportion of false positives (Type I eror) among rejected tests or hypotheses. It is more string ent than other mutiple testing corrections and thereby, will allow very few false positives. 